\title{
Underwater vehicles: The minimum time problem
}

\author{
M. Chyba \\ Department of Mathematics \\ 2565 McCarthy Mall \\ University of Hawaii, Honolulu, HI 96822 \\ Email: mchyba@math.hawaii.edu \\ H. Sussmann \\ Department of Mathematics \\ Rutgers University, Piscataway, NJ 08854 \\ Email: sussmann@hilbert.rutgers.edu \\ H. Maurer, G. Vossen \\ Institut für Numerische Mathematik \\ Westfälische Wilhelms-Universität Münster \\ Einsteinstrasse 62, D-48149 Münster, Germany \\ Email: maurer@math.uni-muenster.de
}

\begin{abstract}
We consider the minimum time problem for a class of underwater vehicles. We focus on the situation of initial and final configurations at rest satisfying $x_{0} \neq x_{f}, z_{0}=$ $z_{f}, \theta_{0}=\theta_{f}=0$. We supplement our theory with a numerical study of optimal bang-bang and singular solutions and include a discussion on a possible Fuller-like phenomenon.
\end{abstract}

\section{INTRODUCTION}

In this paper we pursue the analysis of the minimum time problem for a special class of underwater vehicles, see [5], [6] for previous works. More precisely, the fully actuated controlled mechanical system we consider here describes the equations of motion for an ellipsoidal, neutrally buoyant, uniformly distributed vehicle submerged in an infinitely large volume of incompressible, irrotational and inviscid fluid at rest at infinity. Moreover, we restrict the vehicle to move in the plane $(x, z)$ and we assume it is fully actuated by the use of thrusters. Boundedness on the power of each thruster is assumed.

The primary goal is to understand the structure of the time optimal trajectories. Our computations are based on the Pontryagin maximum principle. Many questions have to be answered: local and global bounds on the number of switchings, optimality of singular arcs, etc. In [6] we study the 2-singular extremals while their optimality is discussed in [5]. We focus here our attention on the following special situation of initial and final configurations both at rest: $x_{0} \neq$ $x_{f}, z_{0}=z_{f}, \theta_{0}=\theta_{f}=0$.

We conjecture that in this case an optimal trajectory must contain totally bang arcs as well as $u_{3}$-singular arcs.

The theoretical conjectures are supported by a numerical study of optimal solutions for different values of the moment of inertia $I$. For higher values of $I$ we obtain

Research supported in part by NSF grant DMS-030641 a purely bang-bang control in all three components with a large total number of switching times. Specifically, for $I=2$ we get a total number of 9 switching times. The structure of the bang-bang control is determined by applying nonlinear programming techniques to the discretized control problem using a fine grid. We then proceed to a refinement of the solution by directly optimizing the switching times. This approach enables us to perform a numerical test of second-order sufficient conditions [1], [2], [11], [12], [13] which shows that the computed solution is indeed a strong local minimum. For a smaller and more realistic value of $I$ (here $I=0.12$ ), the nonlinear programming approach with 10.000 grid points yields a rather complicated combination of bang-bang and singular arcs that support our hypothesis of a chattering control exhibiting a Fuller-like phenomenon [9], [14]. However, optimality of the computed bang-singular solution cannot be shown since applicable sufficient optimality conditions are not available in the literature.

Clearly our assumptions in the model are idealized to a high degree and we cannot draw any practical conclusion from our results. However, our simplified model raises challenging theoretical and numerical questions and could be a good starting point to study a more complete model taking into account viscous effects.

\section{THE MODEL}

For more details on the model of underwater vehicles we consider here, see for instance [5]. We restrict our underwater vehicle to motions in a plane. The variables $x$ and $z$ denotes respectively the absolute horizontal and vertical positions of the vehicle, while $\theta$ represents its orientation. The horizontal and vertical velocities of the vehicle in the body frame coordinates are denoted $v_{1}, v_{3}$. 
$\Omega$ is the scalar angular rate in the plane. We assume the body-fluid mass terms in the body horizontal and vertical directions $m_{1}, m_{3}$ not to be equal, while $I$ is the body-fluid moment of inertia in the plane. Under few assumptions on the fluid and on the vehicle and neglecting viscous effects, the equations of motion for the conservative system are given by

$$
\left(\begin{array}{l}
\dot{x} \\
\dot{z} \\
\dot{\theta} \\
\dot{v}_{1} \\
\dot{v}_{3} \\
\dot{\Omega}
\end{array}\right)=\left(\begin{array}{l}
\cos \theta v_{1}+\sin \theta v_{3} \\
\cos \theta v_{3}-\sin \theta v_{1} \\
\Omega \\
-v_{3} \Omega \frac{m_{3}}{m_{1}} \\
v_{1} \Omega \frac{m_{1}}{m_{3}} \\
v_{1} v_{3} \frac{m_{3}-m_{1}}{I}
\end{array}\right)
$$

We assume the vehicle actuated with thrusters. More precisely, we consider the following inputs: $u_{1}$ is a force in the body 1 -axis, $u_{2}$ is a force in the body 2 -axis and $u_{3}$ is a pure torque in the plane. It follows that the equations of motion of our fully actuated underwater vehicle are described by an affine control system:

$$
\dot{w}=f(w)+\sum_{i=1}^{3} g_{i}(w) u_{i}
$$

where the drift $f$ is given by the equations of motion of the conservative system described above, the $g_{i}$ are the following constant vector fields:

$$
\begin{aligned}
& g_{1}=\left(0,0,0, \frac{1}{m_{1}}, 0,0\right), \\
& g_{2}=\left(0,0,0,0, \frac{1}{m_{3}}, 0\right), \\
& g_{3}=\left(0,0,0,0,0, \frac{1}{I}\right),
\end{aligned}
$$

and the controls $u_{i}$ are measurable bounded functions. Moreover, to reflect the fact that the thrusters have limited power, we assume the following constraints on the inputs:

$$
-1 \leq u_{i} \leq 1, \quad i=1,2,3
$$

Our system belongs to the class of controlled mechanical systems for which a study on the minimum time problem with bounded controls was initiated in [7].

\section{CONJECTURE}

Our previous results are based on the maximum principle which gives necessary conditions for a trajectory to be optimal. For the control system we consider here the Hamiltonian function takes the form:

$$
H(w, \lambda, u)=\lambda^{t} f(w)+\frac{\lambda_{4}}{m_{1}} u_{1}+\frac{\lambda_{5}}{m_{3}} u_{2}+\frac{\lambda_{6}}{I} u_{3}
$$

where the $\lambda_{i}$ 's are the adjoint variables.
From the maximum principle, the adjoint variable are solutions of $\dot{\lambda}=-\frac{\partial H}{\partial w}$ which can be written as:

$$
\begin{aligned}
\dot{\lambda}_{1}= & 0, \\
\dot{\lambda}_{2}= & 0, \\
\dot{\lambda}_{3}= & \lambda_{1}\left(v_{1} \sin \theta-v_{3} \cos \theta\right) \\
& +\lambda_{2}\left(v_{1} \cos \theta+v_{3} \sin \theta\right) \\
\dot{\lambda}_{4}= & -\lambda_{1} \cos \theta+\lambda_{2} \sin \theta-\lambda_{5} \Omega \frac{m_{1}}{m_{3}}-\lambda_{6} v_{3} \alpha, \\
\dot{\lambda}_{5}= & -\lambda_{1} \sin \theta-\lambda_{2} \cos \theta+\lambda_{4} \Omega \frac{m_{3}}{m_{1}}-\lambda_{6} v_{1} \alpha, \\
\dot{\lambda}_{6}= & -\lambda_{3}+\lambda_{4} v_{3} \frac{m_{3}}{m_{1}}-\lambda_{5} v_{1} \frac{m_{1}}{m_{3}},
\end{aligned}
$$

where $\alpha=\frac{m_{3}-m_{1}}{I}$ is a nonzero constant. A solution of the maximum principle is called an extremal. The maximization condition implies that $u_{i-3}(t)=$ $\operatorname{sign}\left(\lambda_{i}(t)\right)$ if $\lambda_{i}(t) \neq 0, \quad i=4,5,6$. In other words, the structure of the time optimal path is governed by the zeroes of the adjoint variables $\lambda_{i+3}=\lambda^{t} g_{i}(w)$. We call $\lambda_{4}, \lambda_{5}, \lambda_{6}$ the switching functions. An extremal is said to be $u_{i}$-bang if the control $u_{i}$ takes it value in $\{-1,+1\}$ for almost every time $t$ and is said to be totally bang if it is $u_{i}$-bang for all controls. A consequence is that an extremal is $u_{i}$-bang if the corresponding switching function does not vanish almost everywhere. If there exists a nonempty interval such that a given switching function is identically zero, no information on the control $u_{i}$ is directly provided by the maximum principle. We then say that the extremal is $u_{i}$-singular on that interval. An extremal is totally singular if it is $u_{i}$-singular for all controls at the same time. A time $t_{s}$ such that $u_{i}$ is not almost everywhere constant on any interval of the form $] t_{s}-\varepsilon, t_{s}+\varepsilon[, \varepsilon>0$, is called a switching time for $u_{i}$.

It is well known that for every fully actuated controlled mechanical system there is no totally singular extremal, see [15]. In [7] the 2-singular extremals are analyzed (i.e. extremals such that 2 controls are singular at the same time) and it is proved that along such extremals there is at most one switching for the nonsingular control. The 2-singular extremals such that the singular controls are identically zero correspond to motions that are horizontal $\left(u_{2}=u_{3} \equiv 0\right)$ or vertical $\left(u_{1}=u_{3} \equiv 0\right)$ translations in body frame coordinates or pure rotations $\left(u_{1}=u_{2} \equiv 0\right)$. Notice that if there is a switching time along a 2-extremal, then the 2 nonsingular controls have to be identically zero. Optimality of these trajectories is discussed in [5]. It is proved that the horizontal translation in body frame coordinates between two configurations at rest with a $u_{1}$-switching at half time is not time optimal. Generalization of this result to vertical translations in body frame coordinates and to pure rotations, both cases for initial and final configurations at rest and a half time switching on the nonsingular controls, uses similar arguments. Notice that the trajectories described above are the fastest between the set of 2-singular extremals joining two positions at rest (indeed it implies that we need at least 
one switching time). Then, a time optimal trajectory between two positions at rest cannot be a 2 -singular extremal. It is an easy verification that the arguments used in [5] still hold without the rest condition on the initial and final configurations. In other words, we have the following.

PROPOSITION 3.1: Horizontal and vertical translations in body frame coordinates as well as pure rotations are not time optimal trajectories. In particular, 2-singular extremals with one switching time on the nonsingular control are not time optimal.

REMARK 3.2: Since we study the problem of joining two positions at rest a control has to be either identically zero or has to contain at least one switching. It follows that an optimal trajectory between two positions at rest cannot be a 2-singular extremal or be formed by concatenations of 2-singular extremals. The simulations show an optimal trajectory that does not contain any 2 -singular arc.

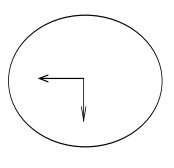

final position

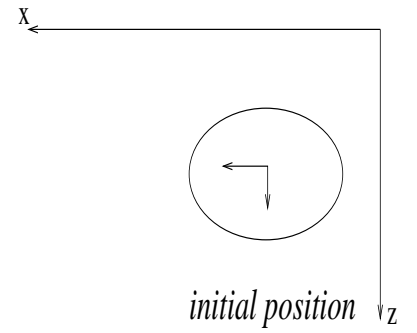

Fig. 1. Initial and Final configurations

Let us now restrict to the situation when the initial and final configurations are respectively $w_{0}=(0,1,0,0,0,0)$ and $w_{f}=\left(x_{f}, 1,0,0,0,0\right)$, see Fig. 1. Numerical solutions are presented in Section IV. From Remark 3.2 we know that an optimal trajectory for this choice of configurations is not a concatenation of 2-singular arcs. We then have to find a way to go faster. Let us assume for the next computations that the bounds on the control $u_{3}$ are very big, in other words assume we can apply a pure torque almost as big as we want. Now, instead of restricting ourself to an horizontal motion in body fixed frame using only full power on the force in the 1-axis direction we assume the vehicle to be rotate in such angle so that we use both forces in the 1 and 2 -axis direction. Let us compute this angle and show that we go faster. Since we do not want any vertical motion along our path, we must satisfy $\dot{z} \equiv 0$ which is, in other words:

$$
\cos \theta v_{3}(t)-\sin \theta v_{1}(t)=0
$$

for each $t$. If we take the maximum value for the two forces $u_{1}, u_{2}$, we get $v_{1}(t)=\frac{t}{m_{1}}$ and $v_{3}(t)=\frac{t}{m_{3}}$ (we focus only on the first half of the trajectory since it is symmetric). It follows that equation (7) becomes $\frac{\cos \theta}{m_{3}}+\frac{\sin \theta}{m_{1}}=0$ which can be written as

$$
\frac{\sin \theta}{\cos \theta}=\tan \theta=\frac{m_{1}}{m_{3}}
$$

(it is obvious that the optimal solution will not be $\frac{\pi}{2}$ ). Using now the fact that the previous equation leads to $\sin \theta=$ $\frac{m_{1}}{\sqrt{m_{1}^{2}+m_{3}^{2}}}$ and that

$$
\dot{x}(t)=\cos \theta v_{1}+\sin \theta v_{3}=t \sin \theta\left(\frac{m_{3}}{m_{1}^{2}}+\frac{1}{m_{3}}\right)
$$

we have:

$$
\dot{x}(t)=t \frac{m_{1}}{\sqrt{m_{1}^{2}+m_{3}^{2}}}\left(\frac{m_{3}}{m_{1}^{2}}+\frac{1}{m_{3}}\right) .
$$

We claim that the coefficient multiplying $t$ in equation (9) is strictly bigger than $\frac{1}{m_{1}}$. Indeed, this coefficient is

$$
\begin{aligned}
& \frac{m_{1}}{\sqrt{m_{1}^{2}+m_{3}^{2}}}\left(\frac{m_{3}^{2}+m_{1}^{2}}{m_{1}^{2} m_{3}}\right) \\
& =\frac{\sqrt{m_{3}^{2}+m_{1}^{2}}}{m_{1} m_{3}}=\frac{1}{m_{1}} \sqrt{1+\frac{m_{1}^{2}}{m_{3}^{2}}}>\frac{1}{m_{1}} .
\end{aligned}
$$

In other words, if $\theta$ is determined such that equation (8) is verified, then we have

$$
\dot{x}(t)>\frac{t}{m_{1}} .
$$

This is the angle at which we can move the fastest along the straight line since it uses the maximum power on both thrusters $u_{1}, u_{2}$. Notice that due to the constraint $\dot{\Omega}=0$ it has to be a strict $u_{3}$-singular arc. It seems reasonable to believe that an optimal trajectory has to be symmetric. Then, since our computations on the previously described $u_{3}$-singular arc hold if $\theta$ is replaced by $\pi-\theta$ a plausible conjecture for an optimal trajectory would be that it is a concatenation of totally bang and strict $u_{3}$-singular arcs as follows. We begin and end with totally bang arcs (to move the vehicle to the right orientation), while a third totally bang arc would connect the two $u_{3}$-singular pieces. The numerical computations, see Section IV, show that for our choice of values of $I, m_{1}, m_{3}$ when the bounds on the control are taken to be equal to one then an optimal trajectory is still formed by totally bang arcs as well as $u_{3}-$ singular arcs while if the bounds are very small then there is no longer a $u_{3}$-singular arc.

\section{NUMERICAL BANG-BANG AND SINGULAR SOLUTIONS}

For numerical computation we choose the values $m_{1}=$ 13.2 and $m_{3}=25.6$ and the boundary conditions $x(0)=$ $0, x\left(t_{f}\right)=2$ and $z(0)=z\left(t_{f}\right)=1$. We study the behavior of the optimal controls w.r.t. the parameter $I$. For higher values of $I$, the optimal controls are bang-bang in all components, whereas for smaller values of $I$, the control component $u_{3}$ contains singular arcs while $u_{1}, u_{2}$ are bangbang. The computations for a wide range of values $I$ show that the optimal control $u_{1}$ is bang-bang with one switching time $t_{f} / 2$ and the control sequence $u_{1}(t)=1 \mid-1$ - Results for the following two cases were obtained by discretizing the optimal control problem and then using the AMPL/LOQO programming and optimization environment [8], [16]. 
Case $I=2$ : Using 1000 grid points and Heun's integration method for ODEs, AMPL/LOQO gives the purely bang-bang controls $u_{2}, u_{3}$ and the corresponding switching functions $\lambda_{5}, \lambda_{6}$ shown in Fig. 2 .
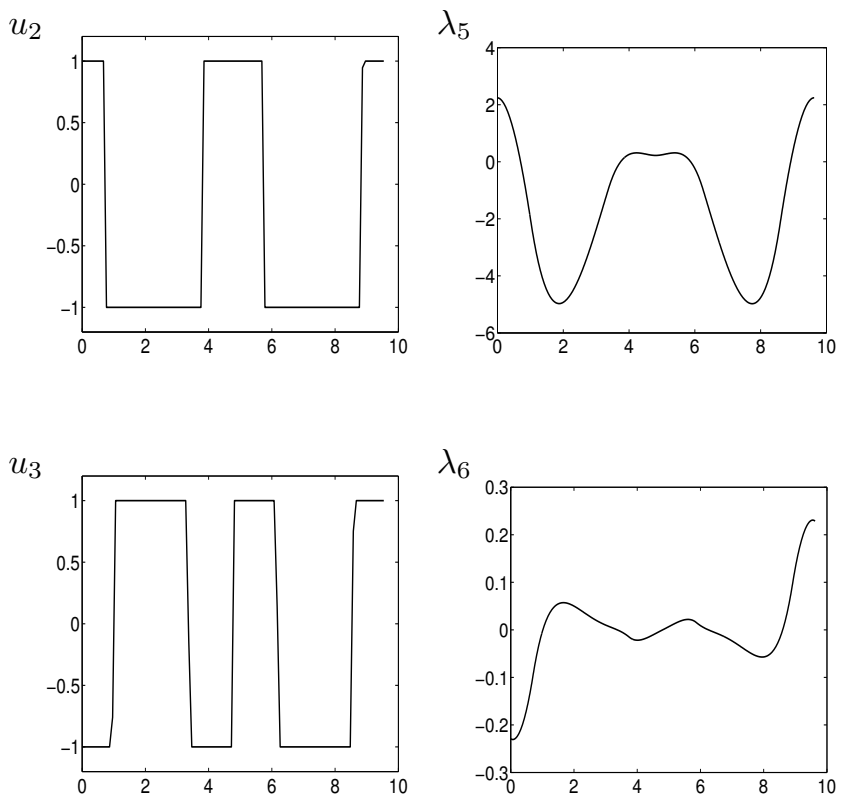

Fig. 2. $\quad I=2$ : optimal controls $u_{2}(t), u_{3}(t)$ and switching functions $\lambda_{5}(t), \lambda_{6}(t)$.
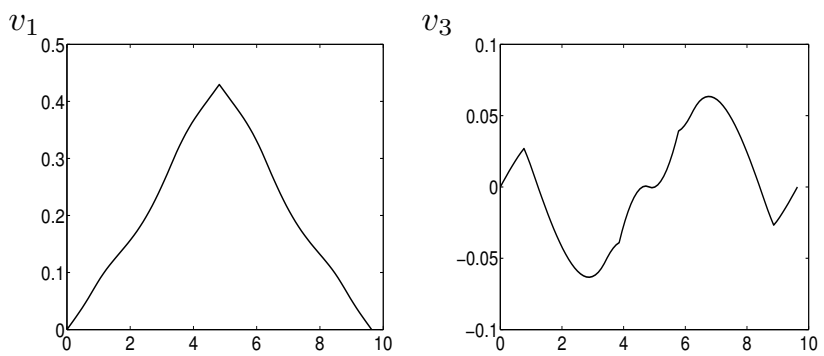

Fig. 3. Velocities $v_{1}(t)$ and $v_{3}(t)$.

Fig. 2 shows that there is a total of 9 switching times for all three control components. To compute the switching times $0=: t_{0}<t_{1}<\ldots<t_{9}<t_{10}:=t_{f}$ and the optimal final time $t_{10}=t_{f}$ with higher precision than the grid size, we use the control package NUDOCCCS of Büskens [3], [4] in combination with an arc-parametrization method described in Kaya, Noakes [10]. In addition, this approach will allow us to verify the second-order sufficient conditions (SSC) for bang-bang controls which have recently been developed in [1], [12], [13]. The optimal control problem is reformulated as a finite-dim. optimization problem using the optimization vector

$$
z:=\left(\xi_{1}, \ldots, \xi_{10}\right), \quad \xi_{k}:=t_{k}-t_{k-1}, \quad k=1, \ldots, 10,
$$

where $\xi_{k}$ denotes the arc duration of the $k$-th bang-bang arc. The resulting scaled problem (cf. problem formulation
(PM2) in [10], p. 81) is given by

$$
\begin{array}{ll}
\min & t_{f}=\sum_{k=1}^{10} \xi_{k} \\
\text { s.t. } & \dot{w}=\zeta\left(f(w)+g_{1}(w) u_{1}+g_{2}(w) u_{2}+g_{3}(w) u_{3}\right), \\
& \zeta(t):=10 \xi_{k} \text { for } t \in I_{k}:=\left[\frac{k-1}{10}, \frac{k}{10}\right], k=1, . ., 10, \\
& w(0)=(0,1,0,0,0,0), \quad w(1)=(2,1,0,0,0,0) .
\end{array}
$$

The values of $u(t)$ in $I_{k}$, the arc durations $\xi_{k}$ and the switching times $t_{k}$ are listed in Table 1, Fig. 4.

\begin{tabular}{c|ccccc}
$k$ & $\xi_{k}$ & $t_{k}$ & $\left.u_{1}\right|_{I_{k}}$ & $\left.u_{2}\right|_{I_{k}}$ & $\left.u_{3}\right|_{I_{k}}$ \\
\hline 1 & 0.71011826 & 0.71011826 & 1 & 1 & -1 \\
2 & 0.31894166 & 1.02905992 & 1 & -1 & -1 \\
3 & 2.34499990 & 3.37405982 & 1 & -1 & 1 \\
4 & 0.37987736 & 3.75393718 & 1 & -1 & -1 \\
5 & 1.06396020 & 4.81789738 & 1 & 1 & -1 \\
6 & 1.06638530 & 5.88428268 & -1 & 1 & 1 \\
7 & 0.37894378 & 6.26322646 & -1 & -1 & 1 \\
8 & 2.34425230 & 8.60747876 & -1 & -1 & -1 \\
9 & 0.31793702 & 8.92541578 & -1 & -1 & 1 \\
10 & 0.71080287 & 9.63621866 & -1 & 1 & 1
\end{tabular}

Fig. 4. Table1 - Switching times and optimal control structure

The computed initial values for the adjoint variables are

$$
\begin{array}{ll}
\lambda_{1}(0)=2.293213, & \lambda_{2}(0)=-0.000011, \\
\lambda_{3}(0)=0.052879, & \lambda_{4}(0)=10.51066, \\
\lambda_{5}(0)=2.243136, & \lambda_{6}(0)=-0.229126 .
\end{array}
$$

The switching functions $\lambda_{i}(t), i=4,5,6$, are nonzero in the interior of the bang-bang arcs and satisfy the control law $u_{i}(t)=\operatorname{sign}\left(\lambda_{i+3}(t)\right), i=1,2,3$; cf. Fig. 2 . Furthermore, the strict bang-bang property in [1] is satisfied in all switching times $t_{k}$. Both controls $u_{1}$ and $u_{3}$ switch at $t_{5}=t_{f} / 2$ due to symmetrie. Hence, the assumption in [1], [12] that different control components have different switching times is not fulfilled here. However, one may argue that due to symmetry this assumption can be dropped here.

The test for SSC in [1], [2], [11], [12], [13] requires further to compute the Hessian of the Lagrangian associated with the above optimization problem. Then one has to show that the Hessian is positive definite on the kernel of the Jacobian of the terminal constraints. Here the Hessian is a $10 \times 10$-matrix while the Jacobian is $6 \times 10$-matrix. The projected Hessian is a $(4 \times 4)$-matrix that has smallest eigenvalue 0.065 and, hence, is positive definite. Thus the solution in Table 1 provides a strong local minimum. Moreover, based on SSC a sensitivity analysis of optimal solutions and switching times w.r.t. variations of system parameters can be performed along the lines of the approach in [11]. Detailed numerical results for the parametric sensitivity derivatives will be given elsewhere.

Case $I=0.12$ : Here, we use a very fine grid with $10^{4}$ grid points and again Heun's method for 
AMPL/LOQO. The optimal time is computed as 9.254699 while the optimal control candidates $u_{2}(t), u_{3}(t)$ and the corresponding switching functions $\lambda_{5}(t), \lambda_{6}(t)$ are depicted in Fig. 5. A zoom on Fig. 5 shows that the control $u_{3}$ contains in fact five singular arcs. It is interesting to remark that along these arcs, either there is no switching for $u_{1}, u_{2}$ or both switching functions $\lambda_{4}, \lambda_{5}$ have to vanish at the same time. For instance, on the third $u_{3}$-singular arc, there is a $u_{1}$-switching at $t_{f} / 2$ while the switching function $\lambda_{5}$ vanishes (but does not change sign). It can be explained with the adjoint equations. Indeed, it is a consequence of the fact that in the equation for $\dot{\lambda}_{6}$ we have the two terms $\lambda_{4} v_{3} \frac{m_{3}}{m_{1}}-\lambda_{5} v_{1} \frac{m_{1}}{m_{3}}$ while $\lambda_{3}$ is absolutely continuous. A more general result can be stated.

Proposition 4.1: Let $i, j, k$ be all distincts. Let $\gamma$ be a $u_{i}$-singular arc and assume this arc to be $u_{j}, u_{k}$-bang. Then, along $\gamma$ the switching functions corresponding to the bang controls $u_{j}, u_{k}$ have to vanish at the exactly same times.

Notice that if a switching function vanishes it does not imply that there is a switching time for the corresponding control. Another interesting point to notice from the pictures $u_{2}$

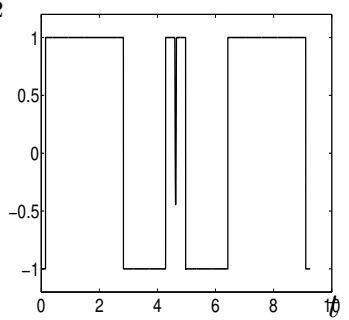

$u_{3}$
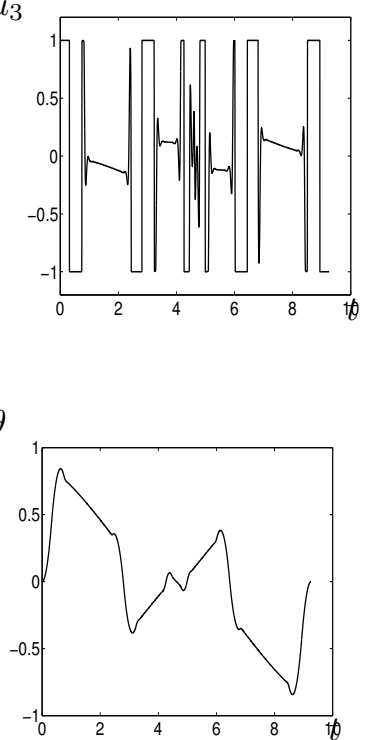

$\lambda_{5}$

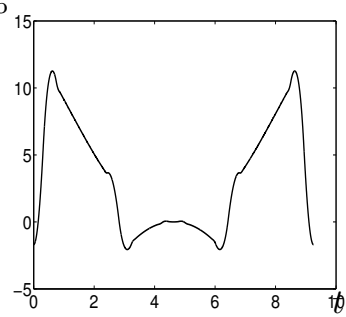

$\lambda_{6}$

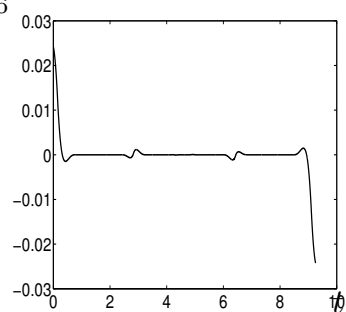

$\Omega$

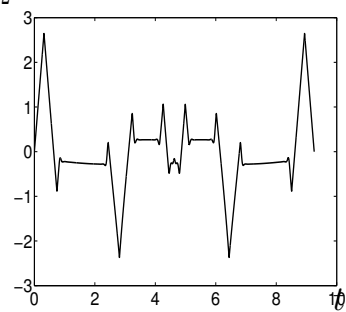

Fig. 5. $I=0.12$ : optimal controls $u_{2}(t), u_{3}(t)$, their switching functions $\lambda_{5}(t), \lambda_{6}(t)$ and $\theta(t), \Omega(t)$.

is that if $\Omega$ is constant then it is along a $u_{3}-$ singular arc.
This could not have happened along a totally bang-bang arc. Indeed if we assume the extremal to be $u_{1}-$ and $u_{2}$-bang, then the condition $\dot{\Omega} \equiv 0$ implies $v_{1} v_{3}\left(m_{3}-m_{1}\right)=-u_{3}$. If $u_{3}$ is \pm 1 , then we must have $v_{1} v_{3}$ constant. Assume $\Omega \equiv 0$. Then, since $u_{1}, u_{2}$ are bang we have $\dot{v}_{1}$ and $\dot{v}_{3}$ constants which implies that $v_{1}$ and $v_{3}$ are linear. Namely, two linear functions such that their product is constant have to be constant. In our case it would mean that $u_{1}$ and $u_{2}$ are zero which is a contradiction. If $\Omega$ is a constant that is not zero we can write the equation for $\frac{d}{d t}\left(v_{1} v_{3}\right)=0$ and replace $v_{1}$ and $\dot{v}_{1}$ by functions depending on $v_{3}$ only. This gives us a polynomial of order 4 for $v_{3}$,

$$
-v_{3}^{4} \Omega \frac{m_{3}}{m_{1}}+v_{3}^{3} \frac{u_{1}}{m_{1}}-v_{3} u_{2} \gamma_{1}+\gamma_{2} \Omega=0,
$$

where $\gamma_{1}, \gamma_{2}$ are nonzero coefficients, which implies that $v_{3}$ has to be constant if $u_{1}$ and $u_{2}$ are.

The numerical optimization results raise another interesting question. It concerns the junction between bang and singular arcs. Fig. 5 suggests that the control $u_{3}$ is chattering at these junctions. This is not surprising since the order of the singular arc is $q=2$ (cf. [14]) which means that along a strict $u_{3}$-singular arc we need to compute the fourth derivative of $\lambda_{6}$ to obtain an expression for the singular control. Moreover, the numerical results show that the strengthened Legendre-Clebsch condition holds and the control is discontinuous at all junctions. Corollary 1 in McDanell and Powers [14] then implies that the control is chattering thus providing another interesting example of a Fuller-like phenomenon. 
Underwater vehicle, Homotopy for $\lambda_{6}, I \in[0.12,2]$
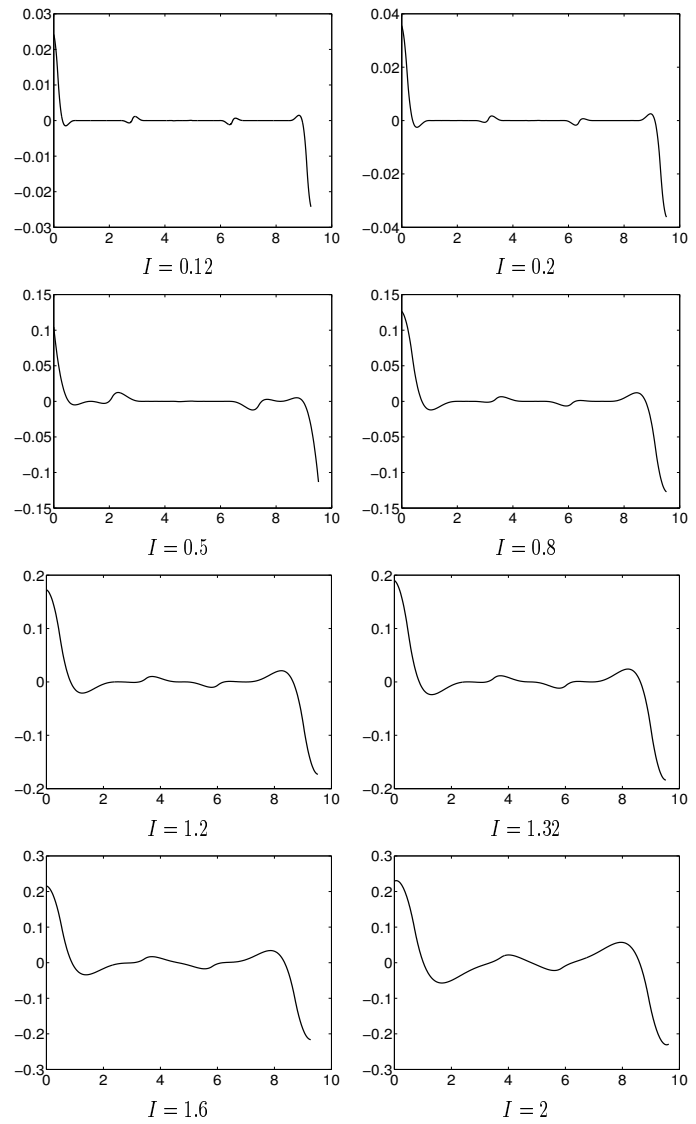

Fig. 6. Homotopy with respect to the value $I$

REMARK 4.2: The simulations show that for each choice of bounds $\alpha_{3}<0<\beta_{3}$ for the control $u_{3}: \alpha_{3} \leq$ $u_{3} \leq \beta_{3}$, there exists a value $I^{*}$ at which we have the transition between totally bang-bang solutions and bangsingular solutions, see Fig. 6. Equivalently, if we fix $I$ and vary the bounds on the control there exists $\alpha_{3}<0<\beta_{3}$ at which the transition occurs. For instance for the value $I=0.12$ the solution is totally bang-bang if we consider the bounds $\left|u_{3}\right| \leq 0.08$ instead of 1 , the transition value being 0.107 . It means that the power we allow on the torque influence the structure of the time-optimal trajectories for our choice of initial and final configurations at rest. Based on the pictures for $\theta$ and $\Omega$ a possible explanation of this phenomenon could be that along a $u_{3}$-singular extremal $\Omega$ needs to be almost constant. This means that $u_{3}$ has to balance the term $v_{1} v_{3}\left(m_{3}-m_{1}\right)$ and if there is not enough power on the torque it is impossible.

\section{REFERENCES}

[1] A.A. Agrachev, G. Stefani and P.L. Zezza, Strong optimality for a bang-bang trajectory, SIAM J. Control and Optimization, vol. 41, No. 4, 2002, pp. 991-1014.

[2] C. Büskens, J.-H.R. Kim, H. Maurer and Y. Kaya, Optimization methods for the verification of second-order sufficient conditions for bang-bang controls, submitted

[3] C. Büskens and H. Maurer, Sensitivity analysis and real-time optimization of parametric nonlinear programming problems, in: Online Optimization of Large Scale Systems, M. Grötschel et al., eds., Springer-Verlag, Berlin, 2001, pp. 3-16.

[4] C. Büskens and H. Maurer, SQP-methods for solving optimal control problems with control and state constraints: adjoint variables, sensitivity analysis and real-time control, J. of Computational and Applied Mathematics, vol. 120, 2000, pp. 85-108.

[5] M. Chyba, A suprising non optimal path for underwater vehicle, Proc. of the 42nd IEEE Conf. on Decision on Control, 2003.

[6] M. Chyba, N.E. Leonard and E.D. Sontag, "Optimality for underwater vehicles", in Proc. of the 4Ond IEEE Conf. on Decision on Control, pp. 4204-4209, 2001.

[7] M. Chyba, N.E. Leonard and E.D. Sontag, Singular trajectories in multi-input time-optimal problems. Application to controlled mechanical systems, Journal on Dynamical and Control Systems, vol. 9 (1), pp. 73-88, 2003.

[8] R. Fourer, D.M. Gay and B.W. Kernighhan, AMPL: A Modeling Language for Mathematical Programming, Duxbury Press, BrooksCole Publishing Company, 1993.

[9] A.T. Fuller, Study of an optimum nonlinear control system, $J$. Electronics Control, vol. 15, 1963, pp. 63-71.

[10] C.Y. Kaya and J.L. Noakes, Computational method for time-optimal switching control, J. of Optimization Theory and Applications, vol. 117, 2003, pp. 69-92.

[11] J.-H.R. Kim and H. Maurer, "Sensitivity analysis of optimal control problems with bang-bang controls", in Proc. of the 42nd IEEE Conf. on Decision and Control, Maui, USA, Dec. 2003, pp. 3281-3286.

[12] H. Maurer and N.P. Osmolovskii, Quadratic sufficient optimality conditions for bang-bang control problems, Control and Cybernetics, vol. 33, 2003, pp. 555-584.

[13] H. Maurer and N.P. Osmolovskii, Second order sufficient conditions for time-optimal bang-bang control problems, SIAM J. Control and Optimization, vol. 42, 2004, pp. 2239-2263.

[14] J.P. McDanell and W.F. Powers, Necessary conditions for joining optimal singular and nonsingular subarcs, SIAM J. on Control, vol. 9, 1971, pp. 161-173.

[15] E.D. Sontag and H.J. Sussmann, "Time-optimal control of manipulators", in Proc. IEEE Int. Conf. on Robotics and Automation, 1986, pp. 1692-1697.

[16] R.S. Vanderbei and D.F. Shanno, An interior point algorithm for nonconvex mathematical programming, Comp. Optim. Appl., vol. 13, 1999, pp. 231-252. 